\title{
Fuentes de estrés en jugadoras élite de balonmano en una competencia nacional
}

\section{Stress Sources in Handball Elite Players in a National Competition}

\author{
Zamora Salazar, Alondra Stefanía ${ }^{1}$, Cosío Ávila, Carlos Alejandro', Ruiz Campos, \\ Jorge Leonardo ${ }^{1}$, Salazar, Ciria Margarita ${ }^{1}$ y Andrade Sánchez, Adriana Isabel ${ }^{1}$.
}

\section{Original}

${ }^{1}$ Facultad de Ciencias de la Educación, Universidad de Colima, México

\section{Resumen}

Objetivo: Determinar el nivel de estrés al que se someten las deportistas de balonmano en una competencia a nivel nacional y observar las fuentes que lo ocasionan.

Método: Se efectuó un estudio descriptivo transversal. Se utilizó el instrumento Fuentes, Síntomas y Estrategias de Afrontamiento al Estrés (FUSIES)) en 60 jugadoras élite de balonmano entre los 13 y 37 años. Participaron en el Campeonato Nacional 2019.

Resultados: Entre los principales resultados, se evidencia que la fuente estresora con calificaciones de extremos más altos, son los estresores académicos-laborales injusticia de autoridades $(\mathrm{M}=3.98 \pm 1.06)$, no tener vacaciones $(\mathrm{M}=3.35 \pm 1.28)$ y falta de tiempo $(\mathrm{M}=3.35 \pm 1.28)$, en los estresores interpersonales-familiares se encuentra no tener dinero $(\mathrm{M}=3.50 \pm 1.28)$, perdida de algo $(\mathrm{M}=3.22 \pm 1.22)$, problemas familiares $(\mathrm{M}=3.17 \pm 1.29)$ y pelear/discutir $(\mathrm{M}=3.17 \pm 1.29)$, en estresores ambientales se localiza calor excesivo $(\mathrm{M}=3.52 \pm 1.86)$, desorden $(\mathrm{M}=3.40 \pm 1.34)$, mucho tráfico $(\mathrm{M}=3.40 \pm 1.25)$, falta de transporte $(\mathrm{M}=3.40 \pm 1.21)$ y ruido excesivo $(\mathrm{M}=3.32 \pm 1.29)$ y en otros estresores menstruación $(\mathrm{M}=3.35 \pm 1.24)$, estar enfermo $(\mathrm{M}=3.10 \pm 1.28)$ y desvelarse $(\mathrm{M}=3.08 \pm 1.22)$.

Conclusiones: La habilitación en estrategias para el afrontamiento del estrés debe ser uno de los elementos relevantes en la planeación deportiva. Las determinantes del estrés deben ser entrenados durante la pretemporada, temporada y pre-competencia para evitar que los estresores amenacen el rendimiento deportivo de las jugadoras.

Palabras clave: fuentes de estrés, balonmano, estrés, deporte élite.

\section{Abstract}

Objective: To determine the level of stress to which handball athletes are subjected in a national competition and observe the sources that cause it.

Method: A descriptive cross-sectional study was carried out. The instrument Sources, Symptoms and Stress Coping Strategies (FUSIES) was used in 60 elite handball players between 13 and 37 years old. They participated in the 2019 National Championship.

Results: Among the main results it is evident that the stressor source with the highest extreme scores are academic-labor stressors, injustice of authorities $(\mathrm{M}=3.98 \pm 1.06)$, not having vacations $(\mathrm{M}=3.35 \pm 1.28)$ and lack of time. $(\mathrm{M}=3.35 \pm 1.28)$, in the interpersonal-family stressors it is found not having money $(\mathrm{M}=3.50 \pm 1.28)$, loss of something $(\mathrm{M}=3.22 \pm 1.22)$, family problems $(\mathrm{M}=3.17 \pm 1.29)$ and fighting / argue $(\mathrm{M}=3.17 \pm 1.29)$, in environmental stressors excessive heat is located $(\mathrm{M}=3.52 \pm$ 1.86), disorder $(\mathrm{M}=3.40 \pm 1.34)$, heavy traffic $(\mathrm{M}=3.40 \pm 1.25)$, lack of transportation $(\mathrm{M}=3.40 \pm 1.21)$ and excessive noise $(\mathrm{M}=3.32 \pm 1.29)$ and in other menstruation stressors $(\mathrm{M}=3.35 \pm 1.24)$, being sick $(\mathrm{M}=3.10 \pm 1.28)$ and staying awake $(\mathrm{M}=3.08$ $\pm 1.22)$.

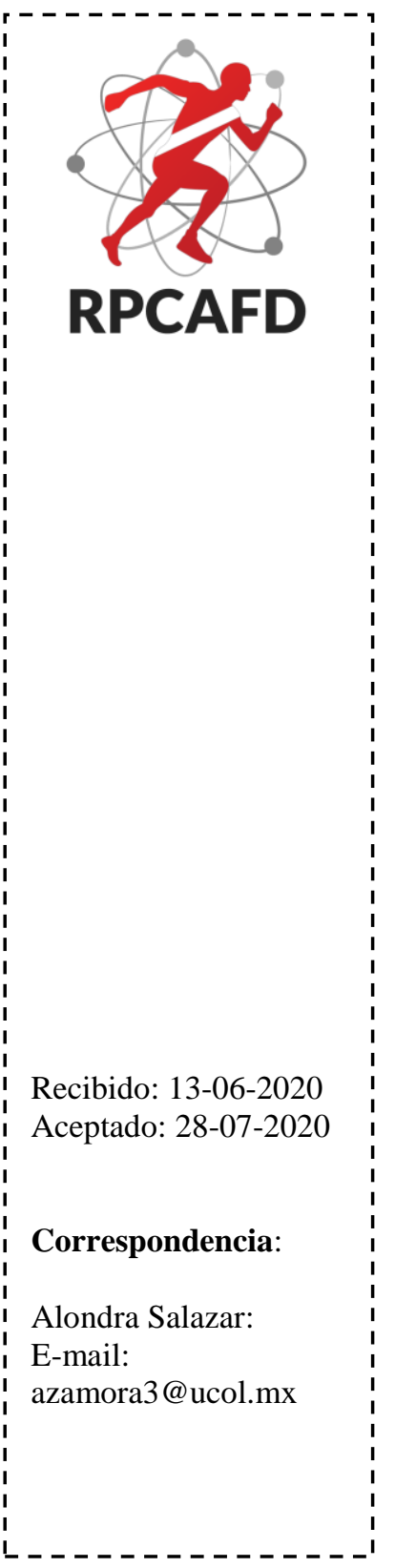

1083 
Conclusions: Enabling strategies for coping with stress should be one of the relevant elements in sports planning. The determinants of stress must be trained during the preseason, season and pre-competition to avoid stressors threatening the sports performance of the players.

Key words: sources of stress, handball, stress, elite sport.

\section{Introducción}

La relación del estrés y el deporte competitivo, sin duda, es muy estrecha; así, la psicología es la disciplina encargada de su estudio y aplicación. Hoy en día la psicología deportiva es un elemento de alta relevancia en la preparación de un atleta de alta competencia. $\mathrm{Su}$ principal tarea es la optimización de las capacidades mentales (mental training), de los estados de ánimo y de la administración del

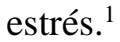

La preparación mental de los deportistas contempla entrenar las habilidades que permitan administrar el estrés, desde la identificación de los estresores hasta la elección de las técnicas somáticas y cognitivas. ${ }^{1,2}$

El deportista experimenta estrés al estar en una situación que le cuesta trabajo manejar antes, durante y después de las competencias. ${ }^{3}$ Puede considerarse como un estado producido por una serie de demandas inusuales que amenazan el bienestar o integridad de la persona, ${ }^{1}$ en el caso del deporte, estas demandas pueden ser familiares, ambientales, técnicodeportivas, o bien, de logística y afectar el desempeño del deportista. ${ }^{2}$

El estrés es una respuesta adaptativa a eventos considerados como amenazas, esto lo experimenta un deportista cuando se le presenta una situación que le cuesta trabajo manejar; lo cual le causa agotamiento, deterioro gradual del organismo. ${ }^{3}$ Las situaciones estresantes durante el desarrollo de las competencias pueden llegar a producir una falta de concentración, la pérdida del foco atencional o el incremento de la ansiedad, la frecuencia cardiaca y/o la tensión muscular. ${ }^{4}$

El atleta, al estar entrenado, podrá lidiar con el estrés que generan las tres demandas básicas (demostración, comparación y evaluación); ${ }^{1,2}$ o bien, podrá ser agobiado por ellas, y perder el control y la concentración antes, durante y después de cada competencia. ${ }^{3}$

El estrés se va produciendo cuando el atleta interpreta una situación como estresante; como un ejemplo puede ser la crítica del propio entrenador, comentarios del equipo contrario o de los espectadores, la aparición de lesiones o cometer errores durante la competencia, inclusive, de los mismos compañeros de equipo, así como de la presión del propio equipo por el óptimo cumplimiento de su función como jugador. ${ }^{2,7}$

De esta forma, se busca analizar las fuentes del estrés deportivo de jugadoras mexicanas participantes de la categoría élite, datos que son relevantes para la toma de decisiones en la planeación del entrenamiento y formación deportiva de las atletas.

\section{Metodología}

\section{Tipo de estudio y muestra}

El diseño del estudio corresponde al de tipo descriptivo transversal, ${ }^{8}$ que permitió describir y caracterizar las "fuentes de estrés", y de cuatro subcategorías de fuentes: estresores académicos-laborales, interpersonalesfamiliares, ambientales y otros estresores ${ }^{9}$.

La muestra participante corresponde al tipo no probabilístico por conveniencia, ${ }^{8,10}$. Este tipo es utilizado por su economía de tiempo, contexto y oportunidad para recolectar la información oportuna de los voluntarios. El tipo de población por conveniencia permite seleccionar aquellos casos accesibles y proximidad de los sujetos que permitan ser incluidos en la investigación. ${ }^{10}$

La muestra se integra de 60 jugadoras mexicanas de balonmano entre los 13 y 23 años, pertenecientes a nueve selectivos estatales, participantes en el Campeonato Nacional Premier de Balonmano de México. Los estados de origen de las informantes son: Aguascalientes, Guanajuato, Sonora, Zacatecas, Jalisco, Colima, Chihuahua, Baja California; así como del Instituto Politécnico Nacional.

\section{Técnicas y procedimientos}

Para el levantamiento de la información, se aplicó la técnica de encuesta y empleó el cuestionario FUSIES $^{9,11,12}$; fue creado en 2010 y validado con 1630 estudiantes (686:H y 944:M) de 18 a 28 años, universitarios de Colima (muestra no aleatoria intencional de 35\% de universitarios de las carreras de Psicología, Pedagogía, Telemática, Medicina, Comunicación, Administración, Ciencias de la Educación, Contabilidad, Derecho y Lenguas 
Extranjeras), se obtuvo el Coeficiente Alpha de Cronbach del FUSIES completo de .9597 , en el 1 er. bloque de .9161 , en el 2do. bloque de .9525 y en el 3er. bloque de .8529; determinándose que es un instrumento confiable, y cuenta con adecuados niveles de estabilidad en su evaluación

Para efectos del trabajo, sólo se utilizó el bloque de estresores, que evalúa 45 fuentes en la escala de 1 (nada) a 5 (extremo) dividido en sub-bloques: estresores académicoslaborales, interpersonales-familiares, ambientales y otros estresores. ${ }^{2,9,11,12}$ Los otros dos bloques —que abordan síntomas y estrategias - se aplicaron, pero no son parte del presente análisis.

El levantamiento de información se realizó en el marco del Campeonato Nacional Elite, celebrado en el mes de diciembre de 2019 en Colima, México, durante 5 días. Para efectos de la contestación del instrumento, se solicitó a los entrenadores de cada selectivo la autorización y fueron las jugadoras quienes decidieron participar. Tres de los integrantes del estudio tuvieron la responsabilidad de explicar el contenido y la forma de contestarlo.

En lo relativo al procedimiento, ${ }^{8}$ se informó a las participantes: se les explicó sobre el objetivo y la manera de llenar el cuestionario. Posteriormente, se calculó el nivel de estrés de cada muestra, en función del promedio obtenido tanto del bloque de estresores como del de síntomas.

\section{Análisis de datos}

Posterior al levantamiento, se realizó la captura de los datos en el software SPSS, versión 25 , para el análisis, se utilizó estadística descriptiva $^{10}$, media y desviación estándar para cada ítem.

\section{Resultados}

Las variables que caracterizan a las jugadoras de balonmano de elite se observan en la tabla 1.

El promedio de edad es de $22,32 \pm 4,57$ años, con una experiencia en la modalidad de $8,98 \pm 3.86$ años. El promedio general de estrés fue de $2,83 \pm 1,16$ puntos.
Tabla 1. Características de la muestra estudiada

\begin{tabular}{lcc}
\hline Variables & $\mathrm{X}$ & $\mathrm{DE}$ \\
\hline Edad (años) & 22.32 & 4.57 \\
Experiencia (años) & 8.98 & 3.86 \\
Fuentes de estrés (total) & 2.83 & 1.16 \\
\hline
\end{tabular}

Los resultados están ordenados en: estresores académicos-laborales, interpersonales-familiares, ambientales y otros estresores. Con relación a los niveles de estrés se muestra $1=$ "nada" y $5=$ "extremo". Los cuatro ítems más relevantes con rango "extremo" dentro de la sección de estresores académico-laborales son: No tener vacaciones $(\mathrm{M}=3.35 \pm 1.28)$, injusticia de autoridades $(\mathrm{M}$ $=3.98 \pm 1.06)$ y falta de tiempo $(\mathrm{M}=$ $3.35 \pm 1.28)$ y llegar tarde $(\mathrm{M}=3.28 \pm 1.19)$ (ver tabla 2).

Las menciones destacadas dentro de la sección 2 de estresores interpersonalesfamiliares son: No tener dinero $(\mathrm{M}=$ $3.50 \pm 1.28)$, pérdida de algo $(\mathrm{M}=3.22 \pm 1.22) \mathrm{y}$ pelear/discutir $(\mathrm{M}=3.17 \pm 1.29)$ y problemas familiares $(\mathrm{M}=3.17 \pm 1.29)$. Podemos tomar estos resultados como valores considerables, en el extremo de la escala (ver tabla 3 ).

Tabla 2. Estresores académico-laborales.

\begin{tabular}{lc}
\hline Ítems & Media \pm DE \\
\hline Impuntualidad & $2.93 \pm 1.11$ \\
Trabajo en exceso & $2.92 \pm 1.13$ \\
\hline Llegar temprano & $2.22 \pm 1.20$ \\
\hline La rutina & $2.78 \pm 1.19$ \\
\hline Llegar tarde & $3.28 \pm 1.19$ \\
\hline Utilizar computadora & $2.30 \pm 1.16$ \\
\hline Ser evaluado & $2.65 \pm 1.24$ \\
No tener vacaciones & $3.35 \pm 1.28$ \\
Aprender otro idioma & $2.85 \pm 1.28$ \\
La figura de autoridad & $2.60 \pm 1.04$ \\
\hline Injusticia de autoridades & $3.98 \pm 1.06$ \\
Relación con compañeros & $2.67 \pm 1.23$ \\
\hline Hablar en público & $2.82 \pm 1.46$ \\
\hline Falta de tiempo & $3.35 \pm 1.28$ \\
\hline
\end{tabular}


Tabla 3. Estresores interpersonales-familiares

\begin{tabular}{lc}
\hline Ítems & Media \pm DE \\
\hline Ser regañado & $2.75 \pm 1.09$ \\
\hline Problemas familiares & $3.17 \pm 1.29$ \\
Pedir permiso & $2.40 \pm 1.27$ \\
No tener dinero & $3.50 \pm 1.28$ \\
Relación con padres & $2.33 \pm 1.27$ \\
Ser criticado & $2.05 \pm 1.26$ \\
Visita de familiares/amigos & $2.03 \pm 1.02$ \\
Ser contradicho & $2.35 \pm 1.02$ \\
Relación con hermanos & $2.25 \pm 1.33$ \\
Estar solo & $1.67 \pm 0.95$ \\
Separación de alguien & $2.33 \pm 1.16$ \\
Pérdida de algo & $3.22 \pm 1.22$ \\
Pelear/discutir & $3.17 \pm 1.29$ \\
Relación de pareja & $2.40 \pm 1.23$ \\
Ser ignorado & $2.88 \pm 1.41$ \\
\hline
\end{tabular}

Dentro de la sección de estresores ambientales, podemos encontrar cinco ítems con el mayor potencial en la creación de estrés extremo, como: desorden $(\mathrm{M}=3.40 \pm 1.34)$, calor excesivo ( $\mathrm{M}$ $=3.52 \pm 1.86) \mathrm{y}$ falta de transporte $(\mathrm{M}=3.40 \pm 1.21)$, mucho tráfico $(\mathrm{M}=3.40 \pm 1.25)$ y ruido excesivo $(\mathrm{M}$ $=3.32 \pm 1.29$ ). Dentro de los ítems resalta desorden en un nivel extremo; que, llevándolo a situación de cancha, este podría ser perjudicial (ver tabla 4).

\section{Tabla 4. Estresores ambientales}

\begin{tabular}{ll}
\hline Ítems & Media \pm DE \\
\hline Aglomeraciones & $2.97 \pm 1.14$ \\
Mucho tráfico & $3.40 \pm 1.25$ \\
Ruido excesivo & $3.32 \pm 1.29$ \\
Frío excesivo & $2.68 \pm 1.29$ \\
Calor excesivo & $3.52 \pm 1.86$ \\
Imprevistos & $2.98 \pm 1.09$ \\
Falta de transporte & $3.40 \pm 1.21$ \\
Lluvia & $2.43 \pm 1.28$ \\
Días nublados & $1.88 \pm 1.25$ \\
Desorden & $3.40 \pm 1.34$ \\
\hline
\end{tabular}

Como se observa en la última sección, de estresores interpersonales-familiares, los resultados se acomodaron en un orden que representa la importancia de los factores en el nivel de estrés; señalando que "menstruación" $(\mathrm{M}=3.25 \pm 1.24)$ representa un factor muy influyente para la creación de un estado de estrés en la deportista, "estar enfermo" $(\mathrm{M}=3.10 \pm 1.28)$, "desvelarse" $(\mathrm{M}$
$=3.08 \pm 1.22)$ en extremo es un factor para el estrés en el deportista (ver tabla 5).

Tabla 5. Otros estresores

\begin{tabular}{lc}
\hline \multicolumn{1}{c}{ Ítems } & Media $\pm \mathbf{D E}$ \\
\hline Peso corporal & $2.92 \pm 1.21$ \\
Desvelarse & $3.08 \pm 1.22$ \\
Menstruación & $3.25 \pm 1.24$ \\
Estar enfermo & $3.10 \pm 1.28$ \\
Necesitar ejercicio & $2.90 \pm 1.37$ \\
\hline
\end{tabular}

\section{Discusión}

La relación entre la fuente y el estrés es muy delgada y depende de varios factores: académicos, laborales, interpersonales y ambientales; ${ }^{2,9}$ por lo tanto, sin un manejo adecuado o estrategias como alternativas, los deportistas presentan alteración en el comportamiento y su conducta, e, igualmente, aumenta la incertidumbre y manifestaciones somáticas. ${ }^{10}$ En otras palabras: el estrés se hace presente cuando el/la deportista no ha logrado la homeostasis.

El evento deportivo en el que participan las jugadoras de balonmano es la competencia de mayor nivel y con mayor relevancia en el país para ser observado por los equipos profesionales y seleccionadores nacionales. El alto nivel de la competencia, sin duda, pone a prueba la preparación mental y la habilidad para el manejo del estrés ${ }^{13}$ de las jugadoras. Es evidente "en esta muestra de jugadoras" la ausencia de las habilidades de afrontamiento y del entrenamiento mental, ${ }^{1,13,14}$ puesto que se observó altos niveles en ítems de fácil manejo con unos niveles de estrés mayor a 3 puntos.

Al analizar los estresores académicolaborales, el tiempo (participación y espera) es un factor con mucha presencia, por ejemplo: falta de tiempo, llegar tarde y no tener vacaciones. El manejo del tiempo precede al sentimiento de baja responsabilidad o poca concentración, ansiedad y miedo a no tener éxito por las percepciones negativas y factores de la personalidad que influyen en la dilación. ${ }^{18}$ La falta de tiempo habla de la ausencia de una organización y planeación del tiempo por parte de la jugadora de balonmano, generando reacciones que afectan al entrenamiento o su participación en competencia. ${ }^{19}$ En un estudio sobre el estrés laboral "para las mujeres" el tiempo es particularmente más estresante, por la diversidad de tareas que son asignadas a las mujeres por su 
condición de rol social. ${ }^{20} \mathrm{El}$ entrenamiento invisible, en este caso, debe incluir la administración del tiempo con perspectiva de género.

Resalta, también - en este primer bloque de estresores - la injusticia de autoridades como un ítem sobresaliente y con alta predominancia; en los entornos multitudinarios y colectivos, el factor de la justicia es un tema que genera orden y propone la equidad entre todos los integrantes..$^{20}$ En el caso de los equipos deportivos, la competitividad nunca genera equidad, todo lo contrario, crea entornos individualistas y competitivos que promueven la presencia de conflicto entre los participantes, del equipo o del torneo. ${ }^{21}$ Estos resultados coinciden con otros estudios realizados en deportistas y participantes de competencias

En lo que se refiere al estresor interpersonalfamiliar - no tener dinero, pérdida de algoresultaron ser las más altas ponderaciones; y problemas familiares y pelear, segundan la lista de factores que desequilibran a las competidoras. La familia es un sistema constituido por un grupo de personas entretejidas por lazos de parentesco, con funciones y estructura jerárquica, normas, reglas; por tanto, la reacción de cada uno de sus miembros afecta a todos los demás. ${ }^{22}$ Por consiguiente, el afrontamiento que deberán realizar las deportistas en crisis familiares implica esfuerzos comportamentales y/o cognitivos que -como se mencionó anteriormente - pueden entrenarse. ${ }^{22}$ No tener dinero resultó ser un factor estresor alto, ligado a la dependencia familiar y estatus sociodemográfico que influye en el estado de ánimo y en el aumento de la ansiedad. ${ }^{18}$

Los estresores ambientales - como el calor excesivo, tráfico, falta de transporte o ruido excesivo- tienen una afectación sensorial importante $^{14}$ y generan pérdida de equilibro de forma muy rápida, al ser propiciadas por un tercero. Las atletas en situaciones de ruido excesivo llegan a una zona de desesperación muy rápido y creación de estrés extremo; con ello, provoca desconcentración, toma de decisiones erróneas que podrían definir el desarrollo del partido.

El calor excesivo tiene una percepción sensorial inmediata en las jugadoras que no son nativas o están acostumbradas a climas fríos o entrenan en instalaciones climatizadas; esto provoca síntomas de deshidratación y pérdida de la conciencia. Una investigación con deportistas universitarios menciona que es factible anticipar el calor excesivo que se pueda presentar en la competencia; por tanto, el entrenador debe estudiar las condiciones climáticas y trabajarlas en el entrenamiento; ya que, de no ser así, podría impactar en el rendimiento del deportista. ${ }^{13}$

Dentro de otros estresores - tratándose de jugadoras femeninas - podemos llegar a la conclusión de que factores como la menstruación y estar enfermo llevan a crear un nivel de estrés extremo, debido a la incomodidad o la frustración de no poder rendir física y mentalmente como de costumbre, haciendo notar su baja de rendimiento a la hora de los encuentros o una acción decisiva; también, dando a notar "en las mujeres" por el estrés que conllevan estas competencias o previas a estas, el retraso o la falta de la menstruación también pueden implicar un estresor a tomar en cuenta dentro de estos parámetros.

Los cambios periódicos relacionados con el ciclo menstrual afectan en el estado funcional de la atleta y, particularmente, en la capacidad de trabajo; y se puede dar como un hecho que el desempeño deportivo puede variar en las distintas fases del ciclo menstrual. ${ }^{14}$

Las fortalezas encontradas dentro de esta investigación son una amplia gama de posibilidades para su estudio y aplicación en las categorías que se desprenden del balonmano, comenzar a trabajar el manejo de las fuentes de estrés desde las categorías inferiores e irlo llevando a cabo durante todos sus procesos hasta llegar a una categoría de competición elite, con ello los y las jugadoras llegan lo suficientemente preparados y ser capaces de afrontar situaciones demandantes de estrés y salir avante ante la situación.

En el caso de las desventajas, un factor importante fue el número de población que se tomó, dado que por la duración de la competición no se logró recabar en su totalidad las muestras que se tenían proyectadas, otro elemento a considerar son las características sociodemográficas que nos permitieran someter sujetos al estudio, cuestiones de traslado, tiempo, desconocimiento y desconfianza por la investigación fueron objeto para la obtención de una escala menor de datos.

Los expertos en psicología deportiva coinciden en que la combinación de los elementos técnico-tácticos, físicos, fisiológicos y psicológicos en el ámbito deportivo permiten competir de una manera intensa, con un alto ritmo de juego, el afrontamiento de presiones, con mayor efectividad e inteligencia.

En el presente estudio, observamos como los estresores injusticia de las autoridades, calor 
excesivo y la menstruación son determinantes del estrés en jugadoras de alta competencia de la disciplina de balonmano. Lo que significa que el manejo del estrés es un elemento altamente necesario para mantener el equilibrio emocional y mental de las jugadoras de balonmano, y debe estar presente en la fase de la planeación del entrenamiento y en la planeación individualizada de las deportistas.

\section{Referencias}

1. Tamorri, S. Neurociencias y deporte. Psicología deportiva. Procesos mentales del atleta. Vol. 70. Editorial Paidotribo. 2004.

2. Salazar, C. M.; Ruvalcaba, S.L.P. y Moreno, P. J. F. Caracterización del estrés en jóvenes deportistas mexicanos durante una competición. Revista española de educación física y deportes, 2017 (416), 35-50.

3. Cassaretto, M. Estrés y afrontamiento en estudiantes de psicología, Revista de psicología, 2003: 21.2, 363-392.

4. Pereira, J.; Peñaranda, D.; Pereira, P.; Pereira, R.; Quintero, J.; Marín, L.; Cruz-León, E. Niveles de depresión y ansiedad en jugadores masculinos de rugby de primera división en Colombia. Revista Peruana de ciencia de la actividad física y del deporte, 2019 (6) 8-8.

5. Dante De Rosa, J. P. K. Síntomas de estrés precompetitivo en jóvenes deportistas brasileños. Revista de Psicología Del Deporte, 2000, 143-157.

6. Guerrero, C.; Sosa-Correa, M.; Zayas, A. y Guil, R. Regulación emocional en jóvenes deportistas ante situaciones adversas en competición. International Journal of Developmental and Educational Psychology, 2017, 373-384.

7. González, G.; Valdivia-Moral, P.; Cachón, J.; Zurita, F. y Romero, O. Influencia del control del estrés en el rendimiento deportivo: la autoconfianza, la ansiedad y la concentración en deportistas. Retos, 2017, 3-6.

8. Hernández-Sampieri, R. y Torres, C. P. Metodología de la investigación, las rutas cuantitativas, cualitativas y mixtas. México: McGraw-Hill Interamericana Editores. 2018.

9. Pérez, S.; Márquez, V.; García, M.; Solorio, C. Realidad virtual y biofeedback en el manejo del estrés ante el Examen General de Egreso a la Licenciatura (eGel). L. Oblitas (Comp.), Psicología de la salud y enfermedades crónicas. Colombia: psicoM. 2006.

10. Manterola, T. O. Técnicas de muestreo sobre una población a estudio. Int. J. Morphol., 2017, 227-232.

11.Moreno, P.; Pérez, S.; Salazar, C.; López, C.; Barajas, L.; Medina, E. Fuentes, síntomas y estrategias de afrontamiento al estrés-competitivo en nadadores. Revista de psicología del deporte, 2017, 26.2: 199-209.

12.Ruvalcaba, S.; Salazar, C.; Flores-Moreno, J., Barajas, L., Rodríguez, E. Caracterización del estrés en deportistas jóvenes mexicanos durante una competición. En Estrés en universitarios. Casos en México, Argentina, Costa Rica y Chile. 97-112. Universidad de colima. México. 2020.

13.Mellalieus, S. D.; Neil, R.; Hantons, S. y Fletcher, D. Competition stress in sport performers: Stressors experienced in the competition environment. Journal of Sport Sciences, 2009, 27(7), 729-744.

14.Cohn, P. J. An Exploratory Study on Sources of Stress and Athlete Burnout in Youth Golf. The Sport Psychologist, 1990, 4(2), 95-106.

15.Dorantes, C. H. y Matus, G. L. El estrés y la ciudad. Revista del Centro de Investigación. Universidad La Salle, 2002, 5(18),71-77.

16. Vayá, E. J. C.; Egeda, R. B. y Miguel-Tobal, J. El ambiente de tráfico como generador de ansiedad en el conductor: Inventario de situaciones ansiógenas en el tráfico (ISAT). Anuario de psicología/The UB Journal of psychology, 1995, (65), 165-184.

17. Konovalova, E. El ciclo menstrual y el entrenamiento deportivo: una mirada al problema. Rev. U.D.C.A Act. \& Div. Cient. 2003, 16(2): 293-302. 
18.Pardo, D.; Perilla, L. y Salinas, C. Relación entre procrastinación académica y ansiedad-rasgo en estudiantes de psicología. CHPs. 2016, 14(1):31-4

19.Durán, M. Bienestar psicológico: el estrés y la calidad de vida en el contexto laboral. Revista nacional de administración, 2010, 1.1: 71-84.

20.Vera, J. M. Gestión eficaz del tiempo y control del estrés. Esic editorial, España. 2012.

21.Unda, S. Significado psicológico del estrés laboral y del malestar en profesores universitarios. Revista Mexicana de Orientación Educativa, 2014, 11-26.

22.Macías, M.; Orozco, C.; Amarís, M.; Zambrano, J.Estrategias de afrontamiento individual y familiar frente a situaciones de estrés psicológico. Psicología desde el Caribe, 2013, 30.1:123-145.

Conflicto de intereses: La presente investigación no representa y no tiene ningún conflicto de interés.

\section{Agradecimientos:}

Externamos nuestro agradecimiento a la Facultad de Ciencias de la Educación, de la Universidad de Colima por la oportunidad de desarrollar la presente investigación, de igual forma, al Instituto Colimense de Deporte y Asociación Estatal de Balonmano por el otorgamiento de los permisos para la aplicación del instrumento. 